\title{
rhPDGF-BB Promotes Proliferation and Osteogenic Differentiation of Bone Marrow Stromal Cells from Streptozotocin-Induced Diabetic Rats through ERK Pathway
}

\author{
Yanfang Zhao, ${ }^{1,2,3}$ Songmei Zhang, ${ }^{1}$ Deliang Zeng,, Lunguo Xia, ${ }^{4}$ Ashwini Lamichhane, \\ Xinquan Jiang, ${ }^{1,5}$ and Fuqiang Zhang ${ }^{1}$ \\ ${ }^{1}$ Department of Prosthodontics, Ninth People's Hospital Affiliated to Shanghai Jiao Tong University School of Medicine, \\ 639 Zhizaoju Road, Shanghai 200011, China \\ ${ }^{2}$ Department of Stomatology, Shanghai East Hospital Affiliated to Tongji University, Shanghai 200011, China \\ ${ }^{3}$ Shanghai Stomatological Diseases Center, Shanghai 200001, China \\ ${ }^{4}$ Department of Oral and Maxillofacial Surgery, Ninth People's Hospital Affiliated to Shanghai Jiao Tong University School of Medicine, \\ Shanghai 200011, China \\ ${ }^{5}$ Oral Bioengineering Laboratory/Regenerative Medicine Lab, Shanghai Key Laboratory of Stomatology, \\ Shanghai Research Institute of Stomatology, Ninth People's Hospital Affiliated to Shanghai Jiao Tong University School of Medicine, \\ 639 Zhizaoju Road, Shanghai 200011, China
}

Correspondence should be addressed to Xinquan Jiang; xinquanj@hotmail.com and Fuqiang Zhang; dr.zhangfuqiang@hotmail.com

Received 30 April 2013; Revised 30 September 2013; Accepted 27 November 2013; Published 29 January 2014

Academic Editor: Eiichi Hinoi

Copyright (C) 2014 Yanfang Zhao et al. This is an open access article distributed under the Creative Commons Attribution License, which permits unrestricted use, distribution, and reproduction in any medium, provided the original work is properly cited.

\begin{abstract}
Management of nonunion fracture and massive segmental bone defects in diabetes remains a challenging clinical problem. Bone marrow stromal cells (BMSCs) are crucial for bone remodeling and hold promise for bone regeneration. However, we have showed previously that diabetes can affect the proliferation and osteogenic potential of BMSCs adversely and a strategy to attenuate the impaired functions of BMSCs is required. Platelet-derived growth factor-BB (PDGF-BB) plays an important role in bone formation. However, little information is available about its effect on diabetic BMSCs. In this study, BMSCs were isolated from streptozotocininduced diabetic rats. After treatment with recombinant human PDGF-BB (rhPDGF-BB), diabetic BMSCs demonstrated enhanced cell proliferation and osteogenic differentiation based on increased expressions of osteogenic genes (Runx2, alkaline phosphatase, and osteocalcin) and Runx2 protein, as well as upregulated alkaline phosphatase activity and mineralization. Furthermore, blocking extracellular signal regulated kinase (ERK) pathway by inhibitor PD98059 repressed the enhanced proliferation and osteogenic differentiation in diabetic BMSCs induced by rhPDGF-BB. Together, these results indicated that rhPDGF-BB stimulates proliferation and osteogenic differentiation partially through ERK pathway in diabetic BMSCs. Therefore, modulation of diabetic BMSCs could augment BMSCs function affected by diabetes and holds significance for future strategies to treat diabetic bone complications.
\end{abstract}

\section{Introduction}

Type 1 diabetes mellitus is an increasingly prevalent systemic disease in the world. In 2012, the prevalence of diabetes was estimated to be $8.3 \%$ worldwide, representing approximately 371 million people living with diabetes [1]. It is well documented that diabetes could contribute to osteoporosis, decreased bone mineral density, increased risk of fracture, and compromised fracture healing rates and bone repair quality [2-6]. The relative risk for hip fracture was as much as 6-7 times higher in patients with diabetes compared with the nondiabetic population $[3,7]$. However, to date, fracture nonunion and bone defects in diabetes are still challenging in the clinic. 
Bone continuously undergoes a process of self-renewal and repair termed bone remodeling which is involved with bone forming and bone resorbing [8]. Increasing evidence suggests that skeletal abnormalities in type 1 diabetes may partially result from the detrimental effects of diabetes on bone formation [9-11]. Bone marrow stromal cells (BMSCs) are a major source of osteoblasts and are crucial for bone remodeling and repair through direct regeneration such as cell differentiation or maturation, or indirect mechanisms via paracrine effects such as increased vascularization [12]. Besides, BMSCs are easily obtained and expanded in vitro, and they are self-renewing, multipotent stem cells that have the capability of differentiating into osteoblasts, chondrocytes, adipocytes, tenocytes, and myoblasts. As their easy availability and multipotent potential, BMSCs are demonstrated as an attractive candidate for tissue engineering applications, which has been effectively used to enhance bone repair and regeneration [13]. However, we and the other group previously showed that BMSCs derived from streptozotocin-induced diabetic rats exhibited decreased proliferative ability and deficiency in osteogenic differentiation compared with control BMSCs. Also, the above function impairments may be responsible for bone disorders associated with diabetes $[14,15]$. Furthermore, these problems could severely affect cell-based therapies for the treatment of diabetic bone diseases. So it is necessary to priming BMSCs to enhance their affected functions.

Platelet-derived growth factor (PDGF) consisting of five types of dimerism (PDGF-AA, PDGF-BB, PDGF-AB, PDGF-CC, and PDGF-DD) is a potent chemoattractant and mitogen that could promote wound and bone healing [16]. Especially, reduced PDGF expression was observed in the diabetic fracture callus, possibly indicating impaired platelet function/aggregation and a corresponding decrease in cell proliferation [17]. Recombinant human PDGF-BB (rhPDGF$\mathrm{BB}$ ) is FDA-approved for periodontal regeneration [18]. Numerous studies indicated that rhPDGF-BB could enhance proliferation and osteogenesis of diverse cell types, as well as stimulate bone formation in fracture and bone defects models [19-22]. In particular, local administration of rhPDGF-BB could enhance fracture healing in diabetic rats [23].

Although increased proliferation and osteoblast differentiation in many types of cells induced by rhPDGF-BB have been widely reported previously, little information is available about its effect on diabetic BMSCs. In this study, we investigated the influence and underlying mechanisms of rhPDGF-BB involved in the regulation of proliferation and osteogenesis of BMSCs derived from diabetic rats. This information may shed light on the effect of rhPDGF-BB on bone metabolism and its mechanism in counteracting bone disorders in diabetes.

\section{Materials and Methods}

2.1. Recombinant Proteins and Antibodies. rhPDGF-BB was provided by BioMimetic Therapeutics, Inc. (Franklin, TN, USA). The MEK inhibitor PD98059 was purchased from Cell Signaling Technology (Beverly, MA, USA). The monoclonal antibodies used in this study include the following: phosphorylation extracellular signal regulated kinase (p-ERK), total ERK (Cell Signaling Technology, MA, USA), $\beta$-actin (Sigma, St. Louis, MO, USA), and runt-related transcription factor 2 (Runx2) (bioworld, MN, USA).

2.2. Animal Models. All animal experiments were approved by the Animal Research Committee of the Ninth People's Hospital affiliated to Shanghai Jiao Tong University, School of Medicine. Eight 4-week-old male Wistar rats were used in the study. Diabetes was induced via a single intraperitoneal injection of streptozotocin $(65 \mathrm{mg} / \mathrm{kg}$ ) (Sigma, St. Louis, MO, USA) dissolved in $0.01 \mathrm{M}$ citrate buffer $(\mathrm{pH}=4.5)$ as previously described [14]. The diabetic rats were confirmed if their blood glucose concentrations were higher than $16.7 \mathrm{mmol} / \mathrm{L}$ tested by a blood glucose meter (Accu-Chek Perferma, Roche Diagnostics, Indianapolis, IN, USA) 1 week after-injection [24]. The blood glucose concentrations and body weights were measured in the same day before injection, 1 week, and 12 weeks after-injection (data not shown). All animals were fed with a regular diet and water ad libitum.

2.3. Isolation and Culture of Rat BMSCs. After euthanasia, BMSCs were harvested from the tibia and femur bone marrow of diabetic rats and cultured in low glucose Dulbecco's Modified Eagle Medium (DMEM, low glucose; Gibco, Grand Island, NY, USA) supplemented with $10 \%$ fetal bovine serum (Hyclone, Logan, UT, USA), containing $100 \mathrm{U} / \mathrm{mL}$ penicillin, $100 \mathrm{U} / \mathrm{mL}$ streptomycin, and $2 \mathrm{mM} \mathrm{L}$-glutamine (Sigma) as previously described. After BMSCs were incubated for 24 hours, the medium was changed to discard nonadherent cells. Then the medium was refreshed every 3 days. When confluence reached approximately $80 \%$, cells were detached and passaged. Cells at passage 2 or 3 were used for all experiments. To study the osteogenic differentiation of BMSCs, osteogenic medium containing 10-8 $\mathrm{M}$ dexamethasone, $50 \mu \mathrm{g} / \mathrm{mL} \mathrm{L-2-}$ ascorbic acid, and $10 \mathrm{mM} \beta$-glycerophosphate were used.

To examine the effect of rhPDGF-BB on proliferation and osteogenic differentiation of diabetic BMSCs, the cells were divided into 3 groups: control, $10 \mathrm{ng} / \mathrm{mL}$ rhPDGF-BB, and $50 \mathrm{ng} / \mathrm{mL}$ rhPDGF-BB. For analysis of the role of ERK signaling in the aforementioned effect of rhPDGF-BB, the divided groups were as follows: control, $10 \mathrm{ng} / \mathrm{mL} \mathrm{rhPDGF}-$ $\mathrm{BB}$, and $10 \mathrm{ng} / \mathrm{mL}$ rhPDGF-BB + PD98059.

2.4. Cell Proliferation Assay. MTT assay was used to assess the amount of viable cells. Briefly, BMSCs were cultured in 96 -well plates at $3 \times 10^{3}$ cells/well initial density, treated with or without rhPDGF-BB or PD98059. After the indicated culture periods, MTT $(5 \mathrm{mg} / \mathrm{mL})$ was added into each well and incubated for 4 hours at $37^{\circ} \mathrm{C}$. After removing medium, DMSO was used to dissolve formazan and the absorbance value was measured using a microplate reader (Bio-tek, Vermont, USA) at $490 \mathrm{~nm}$.

2.5. Real-Time PCR Analysis. At 7 days, total RNA was extracted from each group using Trizol reagent (Invitrogen, Carlsbad, CA, USA) and was transcribed with PrimeScript 


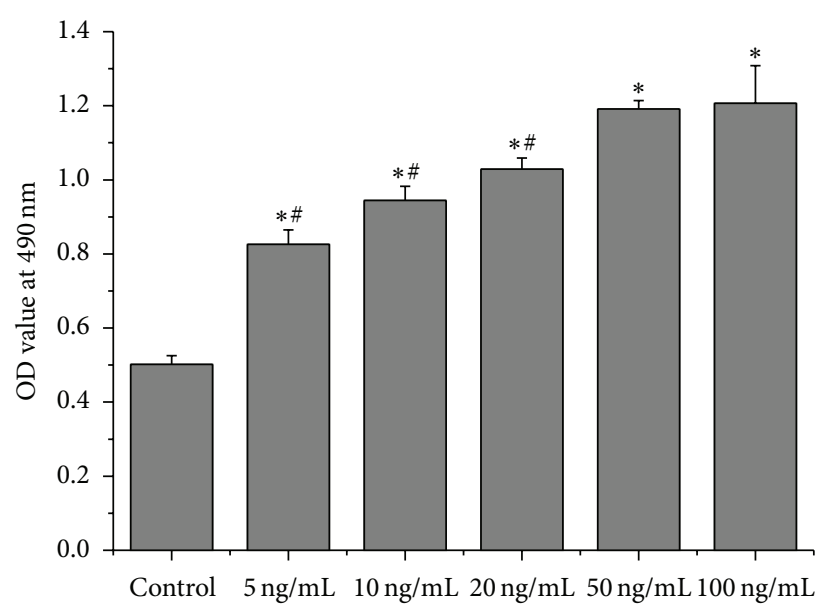

(a)

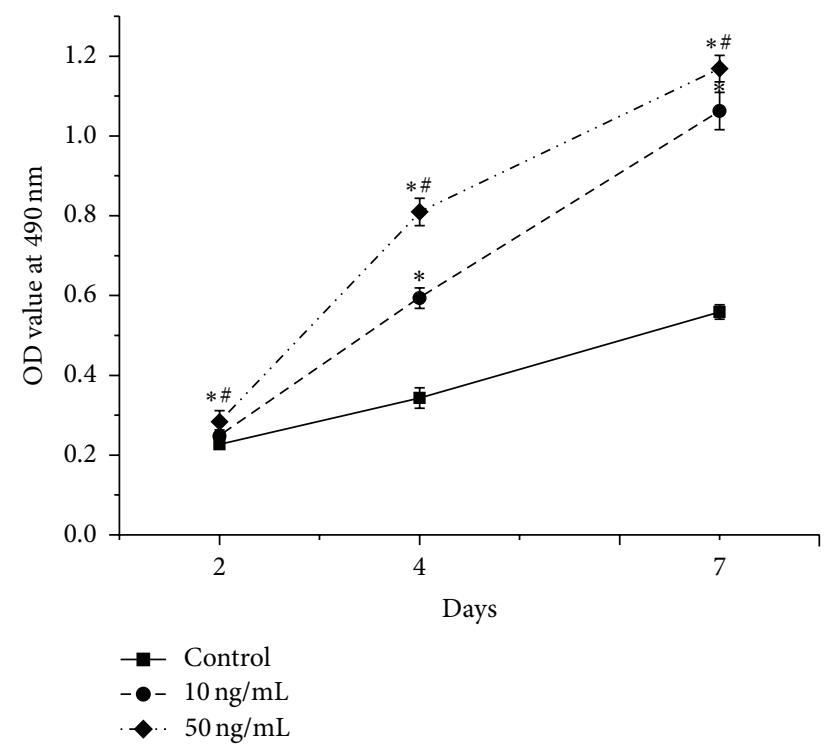

(b)

FIGURE 1: Effects of rhPDGF-BB on proliferation of diabetic BMSCs. (a) MTT assay results of diabetic BMSCs treated with or without rhPDGF-BB (5-100 ng/mL) for 7 days, ${ }^{*} P<0.05$, versus control, and ${ }^{\#} P<0.05$, versus $50 \mathrm{ng} / \mathrm{mL}$. (b) MTT assay results of diabetic BMSCs treated with or without rhPDGF-BB $(10 \mathrm{ng} / \mathrm{mL}$ or $50 \mathrm{ng} / \mathrm{mL})$ for 2,4 and 7 days, ${ }^{*} P<0.05$, versus control, and ${ }^{\#} P<0.05$, versus $10 \mathrm{ng} / \mathrm{mL}$.

RT reagent kit (TaKaRa, Kyoto, Japan). The cDNA amplification and detection was performed with the Bio-Rad iQ5 real-time PCR system (Bio-Rad, Hercules, CA, USA) using SYBR Premix Ex Taq Kit (TaKaRa) and specific primers previously used [14]. Then the relative gene expression level was normalized to the internal control ( $\beta$-actin) based on the $2^{-\Delta \Delta \mathrm{Ct}}$ method.

2.6. Alkaline Phosphatase (ALP) Staining and ALP Activity Assay. Cells were plated in 12-well plates at a density of 2 $\times 10^{4}$ cells/well and incubated for 7 or 14 days. For ALP staining, after being fixed for $15 \mathrm{~min}$ at $4^{\circ} \mathrm{C}$ with $10 \%$ formalin, BMSCs were treated with a BCIP/NBT solution (Beyotime, Shanghai, China) in the dark, and areas stained purple were regarded as positive. Photomicrographs of each group were captured with a light microscope (Olympus). ALP activities were determined using p-nitrophenyl phosphate (sigma) as described previously [25].

2.7. Mineralization Measurement. Cells were plated into a 12-well dish at a density of $2 \times 10^{4}$ cells/well and incubated for 21 days. For alizarin red staining, BMSCs were fixed in $70 \%$ ethanol for $1 \mathrm{~h}$ and stained with $40 \mathrm{mM}$ alizarin red S solution for $30 \mathrm{~min}$ at room temperature. Nonspecific staining was removed by several washes in distilled water. For von Kossa staining, after being fixed in $10 \%$ formalin for $15 \mathrm{~min}$, cells were stained with $5 \%$ silver nitrate and placed under ultraviolet light for $30 \mathrm{~min}$. Then cells were treated with $5 \% \mathrm{NaS}_{2} \mathrm{O}_{3}$ for $2 \mathrm{~min}$ and washed with distilled water [26]. Photomicrographs of each group were captured with a light microscope (Olympus).
2.8. Western Blot Analysis. For Western blotting, different groups of cells were lysated with a protein extraction regent containing protease inhibitor cocktail, phosphatase inhibitor cocktail and phenylmethanesulfonyl fluoride (PMSF) (Kangchen, Shanghai, China). The protein concentration was measured according to the BCA protein assay kit manufacturer's protocol (Beyotime, Shanghai, China). Equal protein samples were separated on SDS-polyacrylamide gel electrophoresis (PAGE) and then electrotransferred to a polyvinylidene difluoride membrane. The membranes were blocked and primary antibodies (rabbit anti-rat Runx2, 1:500; rabbit anti-rat ERK, $1: 1000$; rabbit anti-rat p-ERK, $1: 1000$; mouse anti-rat $\beta$-actin, $1: 1000)$ were incubated. Finally, the blots were visualized with horseradish peroxidase (HRP)-conjugated goat anti-rabbit or anti-mouse IgG $(1: 1000)$ using the ECL plus reagents (Amersham Pharmacia Biotech, Arlington Heights, USA) by an UVItec ALLIANCE 4.7 gel imaging system. The relative integrated density of each protein band was analyzed by NIH image J 1.34s.

2.9. Statistical Analysis. Data were expressed as mean \pm standard deviation (SD). All experiments were replicated at least three times. One-way analysis of variance (ANOVA) was performed with post hoc SNK for multiple comparisons (SPSS Inc., Chicago, USA). And $P<0.05$ was considered to be statistically significant.

\section{Results}

3.1. rhPDGF-BB Promoted the Proliferation of Diabetic BMSCs. The MTT assay was performed to investigate whether rhPDGF-BB has positive effect on the proliferation 
of diabetic BMSCs. Figure 1(a) showed that cell proliferation significantly increased in groups treated with $5-100 \mathrm{ng} / \mathrm{mL}$ rhPDGF-BB compared to negative controls $(P<0.05)$, but there was no significant difference between $50 \mathrm{ng} / \mathrm{mL}$ group and $100 \mathrm{ng} / \mathrm{mL}$ group in OD values. Moreover, Figure 1(b) revealed that cells cultured in $10 \mathrm{ng} / \mathrm{mL}$ and $50 \mathrm{ng} / \mathrm{mL}$ rhPDGF-BB groups grew faster than cells cultured in control group on days 2,4 , and 7 . The above results indicated that rhPDGF-BB promoted proliferation of diabetic BMSCs in a time- and dose-dependent manner.

\section{2. rhPDGF-BB Enhanced the Osteogenic Differentiation of} Diabetic BMSCs. Firstly, real-time PCR was performed to evaluate the expression levels of osteogenic genes including Runx2, ALP, and osteocalcin (OCN) on day 7. Compared to the control group, rhPDGF-BB increased the relative expression of Runx 2 by $78 \%$ and $143 \%$, the ALP by $91 \%$ and $171 \%$, and the OCN by $113 \%$ and $221 \%$, with respect to $10 \mathrm{ng} / \mathrm{mL}$ and $50 \mathrm{ng} / \mathrm{mL}$ PDGF-BB group, respectively (Figure 2(a)). Consistent with increased Runx2 mRNA levels, Runx2 protein level was also increased in $10 \mathrm{ng} / \mathrm{mL}$ and $50 \mathrm{ng} / \mathrm{mL}$ rhPDGF-BB group compared with the control group on day 7 , which was detected by western blot analysis (Figure 2(b)). Moreover, there was significant difference between $10 \mathrm{ng} / \mathrm{mL}$ group and $50 \mathrm{ng} / \mathrm{mL}$ group (Figure 2(b)).

To further evaluate the osteogenic differentiation ability, ALP activity and matrix mineralization ability were analyzed at the defined time points. On days 7 and 14, more intense ALP staining was observed in BMSCs treated with rhPDGF-BB groups than in the control BMSCs, while the most intense ALP staining was observed in the $50 \mathrm{ng} / \mathrm{mL}$ group (Figure 2(c)). Similarly, at days 7 and 14, rhPDGF-BB (10 ng/mL and $50 \mathrm{ng} / \mathrm{mL}$ rhPDGF-BB groups) significantly increased ALP activity compared with the control group in a dose-dependent manner (Figure 2(d)).

In addition, the Alizarin red staining of mineralized bone nodules on day 21 showed that rhPDGF-BB $(10 \mathrm{ng} / \mathrm{mL}$ and $50 \mathrm{ng} / \mathrm{mL}$ rhPDGF-BB groups) significantly increased matrix mineralization compared with untreated group, while the $50 \mathrm{ng} / \mathrm{mL}$ group presented the strongest staining among all groups (Figure 2(e)). Consistent with alizarin red staining results, von Kossa staining also showed the same trend similar to results of Alizarin red staining between the rhPDGF-BB groups and the control group (Figure 2(f)). Taken together, our data revealed that rhPDGF-BB promoted osteogenic differentiation of diabetic BMSCs.

3.3. rhPDGF-BB Activated ERK Pathway in Diabetic BMSCs. To determine how rhPDGF-BB promoted the functions of diabetic BMSCs, we investigated whether ERK pathway in diabetic BMSCs was activated by rhPDGF-BB treatment. The results showed that levels of $\mathrm{p}$-ERK in rhPDGF-BB treated diabetic BMSCs were unregulated significantly (Figure 3(a)). Quantitatively, the p-ERK increased approximately eightfold at $5 \mathrm{~min}$, sevenfold at $15 \mathrm{~min}$, and sevenfold at $30 \mathrm{~min}$ and decreased to twofold at $60 \mathrm{~min}$ in rhPDGF-BB treated group as compared with control group (Figure 3(b)). Western blot analysis indicated that $20 \mu \mathrm{M}$ PD98059 blocked ERK signaling efficiently (Figure 3(b), comparing lane 6 with lane 3).

3.4. rhPDGF-BB-Induced Proliferations of Diabetic BMSCs Was Inhibited by PD98059. We assessed the effect of inhibition of ERK pathway on proliferation induced by rhPDGF-BB in diabetic BMSCs. The MTT results showed that PD98059 could significantly suppress rhPDGF-BB-mediated enhancement of diabetic BMSCs proliferation at days 2, 4, and 7 (Figure 4).

3.5. rhPDGF-BB-Enhanced Osteogenic Differentiation of Diabetic BMSCs Was Repressed by PD98059. To elucidate whether ERK pathway was involved with the rhPDGF-BBenhanced osteogenic differentiation of diabetic BMSCs, we performed real-time PCR, western blot analysis, ALP activity and matrix mineralization analysis.

Real-time PCR results showed that $10 \mathrm{ng} / \mathrm{mL}$ PDGF$\mathrm{BB}$ group significantly increased the expression of Runx2, ALP, and OCN at day 7. Conversely, PD98059 decreased the expression of Runx 2 by $45 \%$, the ALP by $32 \%$, and the OCN by $41 \%$ compared with $10 \mathrm{ng} / \mathrm{mL}$ rhPDGF-BB group (Figure 5(a)). In addition, western blotting demonstrated that PD98059 markedly decreased rhPDGF-BB-enhanced expression of protein Runx2 (Figure 5(b)).

ALP staining was stronger in $10 \mathrm{mg} / \mathrm{mL}$ rhPDGF-BB group than in control group, while PD98059 decreased the intensity of ALP staining compared with $10 \mathrm{mg} / \mathrm{mL}$ rhPDGF-BB group (Figure 5(c)). Meanwhile, rhPDGF-BB significantly increased ALP activity compared with control group, while PD98059 could significantly decrease rhPDGFBB-enhanced ALP activity (Figure 5(d)). Furthermore, the Alizarin red staining showed that rhPDGF-BB significantly increased matrix mineralization compared with the control group, whereas PD98059 could significantly decrease matrix mineralization compared with $10 \mathrm{ng} / \mathrm{mL}$ group (Figure 5(e)). Collectively, these results indicated that ERK pathway is the mediator of the enhanced effect of rhPDGF-BB on osteogenesis of diabetic BMSCs.

\section{Discussion}

Diabetes is associated with a series of bone complications, which are challenging in the clinic. It is well documented that diabetes impaired osseous healing, showing as delayed union or nonunion [27]. Recently, considerable interest has focused on cell-based therapy to promote bone regeneration and repair, even in diabetes [28]. As primitive cell population, bone marrow stromal cells (BMSCs) are attractive cell sources and are crucial for bone remodeling and regeneration. However, recently, BMSCs from diabetic animals have been shown to have reduced proliferation and differentiation capacity, which may be responsible for bone disorders associated with diabetes $[14,15]$. And the aforementioned problems may affect cell-based therapies for treatment of diabetic bone diseases. Therefore, modulation of diabetic BMSCs to enhance their proliferation and functions could have potential clinical implications in diabetes. 


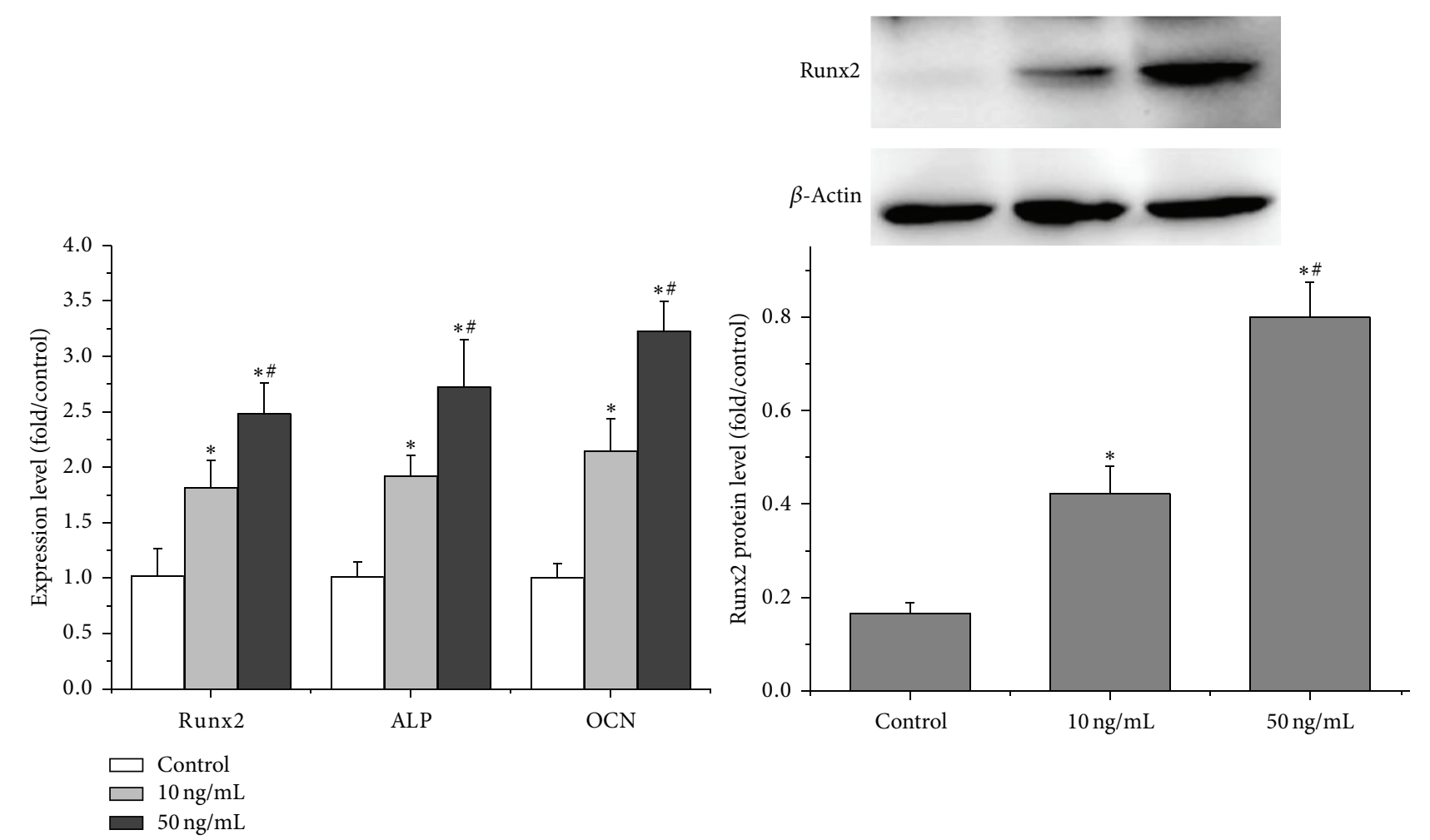

(a)

(b)
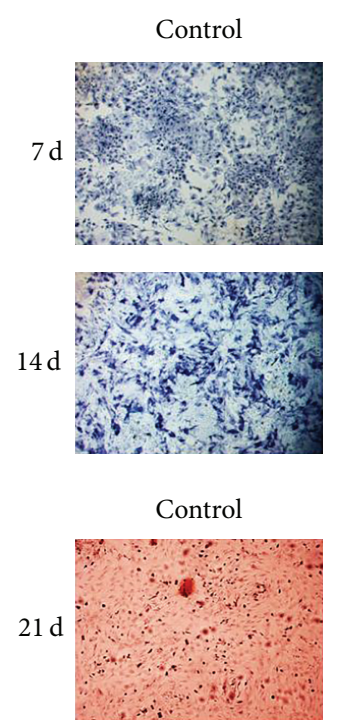

Control

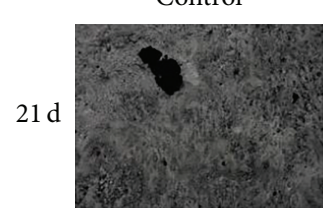

$10 \mathrm{ng} / \mathrm{mL}$
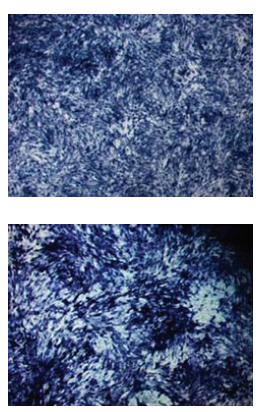

(c)

$10 \mathrm{ng} / \mathrm{mL}$

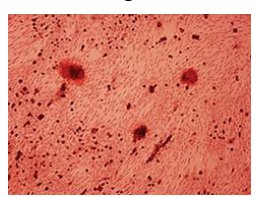

(e)

$10 \mathrm{ng} / \mathrm{mL}$

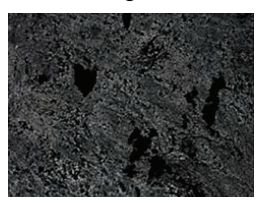

(f)
$50 \mathrm{ng} / \mathrm{mL}$
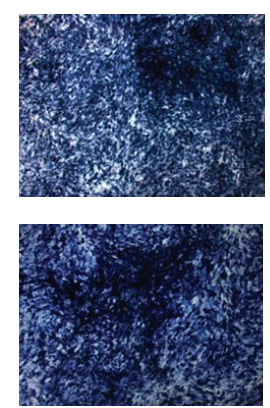

$50 \mathrm{ng} / \mathrm{mL}$

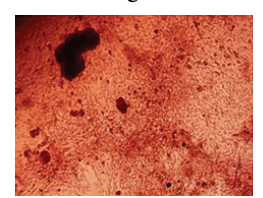

$50 \mathrm{ng} / \mathrm{mL}$

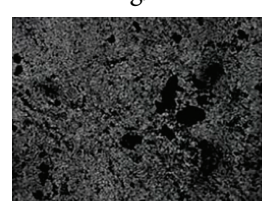

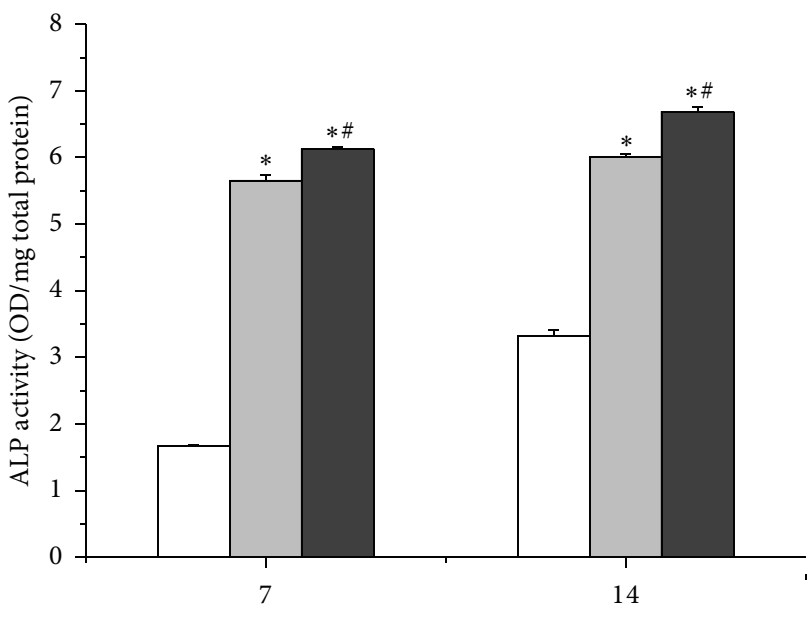

Days

(d)

FIGURE 2: Effects of rhPDGF-BB on osteogenic differentiation of diabetic BMSCs. Cells were treated with or without rhPDGF-BB (10 ng/mL or $50 \mathrm{ng} / \mathrm{mL}$ ) for the indicated periods. (a) Real-time PCR analysis of ALP, Runx2, and OCN mRNA expression. (b) Western blot analysis for the relative Runx2 protein levels. (c) ALP staining on days 7 and 14 (16x). (d) Quantitative results of ALP assay on days 7 and 14. (e) Alizarin red staining for mineralized nodules on day 21 (50x). (f) von Kossa staining for mineralized nodules on day 21 (50x). ${ }^{*} P<0.05$, versus control, and ${ }^{\#} P<0.05$, versus $10 \mathrm{ng} / \mathrm{mL}$. 


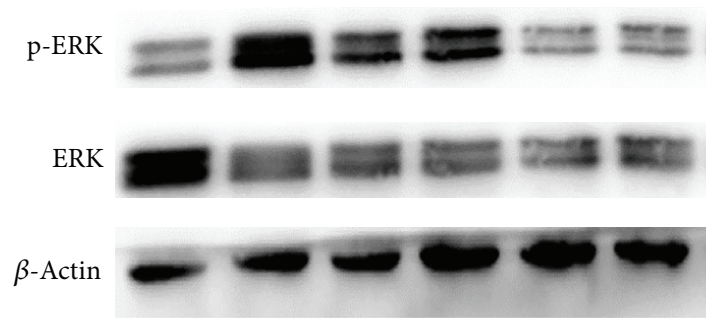

(a)

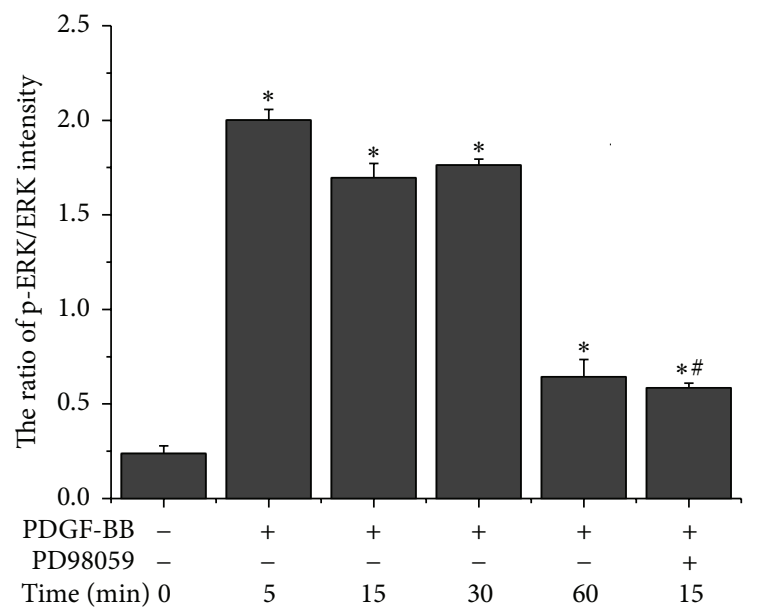

(b)

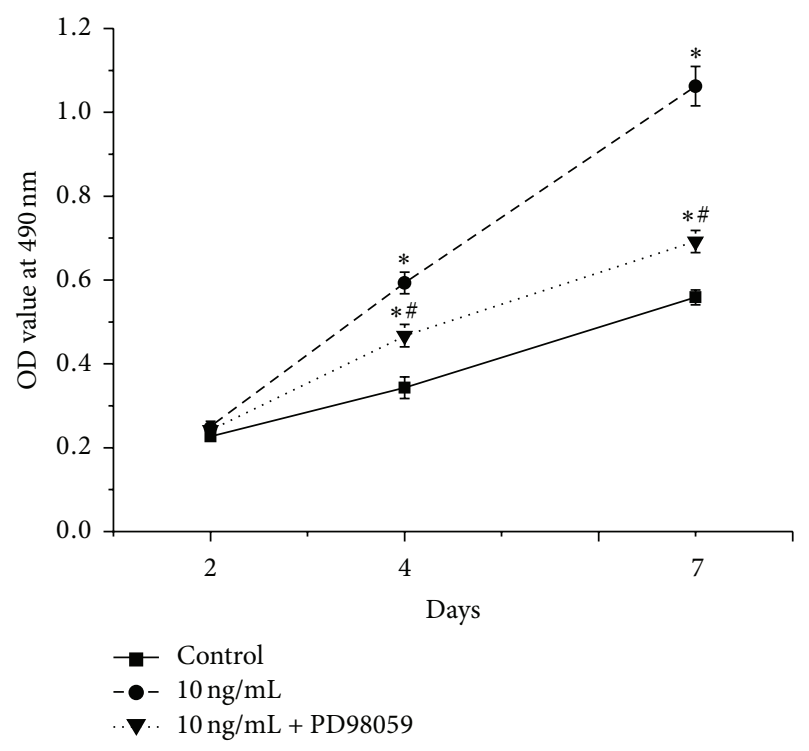

FIGURE 4: rhPDGF-BB-induced cell proliferation was inhibited by suppression of ERK pathway. Cells were pretreated with or without $20 \mu \mathrm{M}$ PD98059 for $1 \mathrm{~h}$ and treated with $10 \mathrm{ng} / \mathrm{mL}$ rhPDGF-BB. The unmanipulated cells were used as controls. MTT assay was assessed on days 2,4 , and $7 .{ }^{*} P<0.05$, versus control, and ${ }^{\#} P<0.05$, versus $10 \mathrm{ng} / \mathrm{mL}$.

the effects of rhPDGF-BB on proliferation and osteoblastic differentiation of diabetic BMSCs.

BMSCs proliferation in response to growth factors is essential for skeletal development, bone remodeling, and fracture repair. The literature suggested that PDGF-BB is potent stimulator of cell growth [33]. Consistently, in this study, MTT assays showed that rhPDGF-BB enhanced proliferation of diabetic BMSCs in a dose- and time-dependent manner. These results were also consistent with our previous observations in normal BMSCs and demonstrated clearly that rhPDGF-BB could improve the diabetes-related proliferative impairment of BMSCs [22].

Our results also demonstrated that rhPDGF-BB significantly upregulated the mRNA levels of the osteogenic genes such as ALP, Runx2, and OCN and the protein levels of Runx2 in a dose-dependent manner. Furthermore, rhPDGFBB increased ALP activity levels and the induction of mineralization of diabetic BMSCs in vitro. Runx2 is an early cell fate marker required for osteogenic differentiation, which could regulate downstream genes maintaining osteoblastic phenotype, such as ALP and OCN $[34,35]$. ALP, a membrane bound enzyme, is a marker of early bone differentiation. OCN is a later stage protein expressed in the mature bone tissue. Altogether, our data indicates that rhPDGF-BB stimulates diabetic BMSCs differentiation and maturation. These data are in accordance with studies using cells from healthy individuals $[22,31]$. These results may help to explain the enhancement of rhPDGF-BB on fracture healing in diabetic rats in a cellular and molecular basis and also suggest that rhPDGFBB could be used as a potent agent in bone disorders 


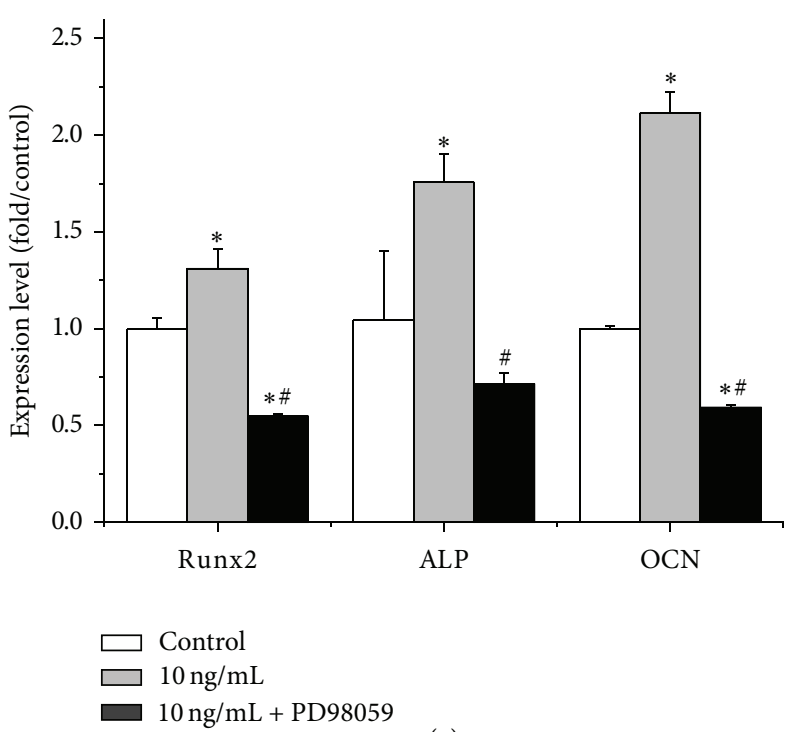

(a)

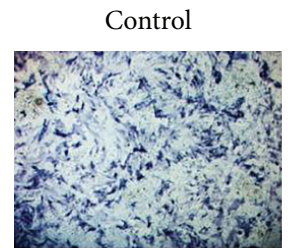

Control
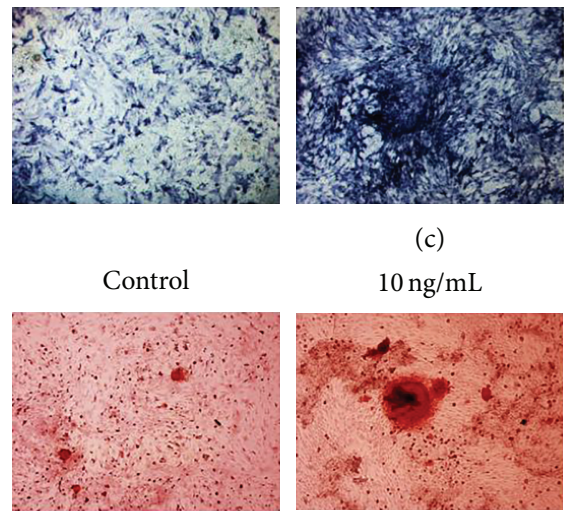

(c)

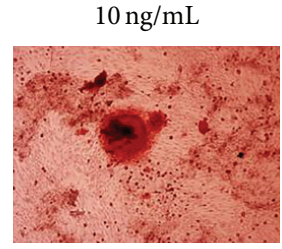

(e)

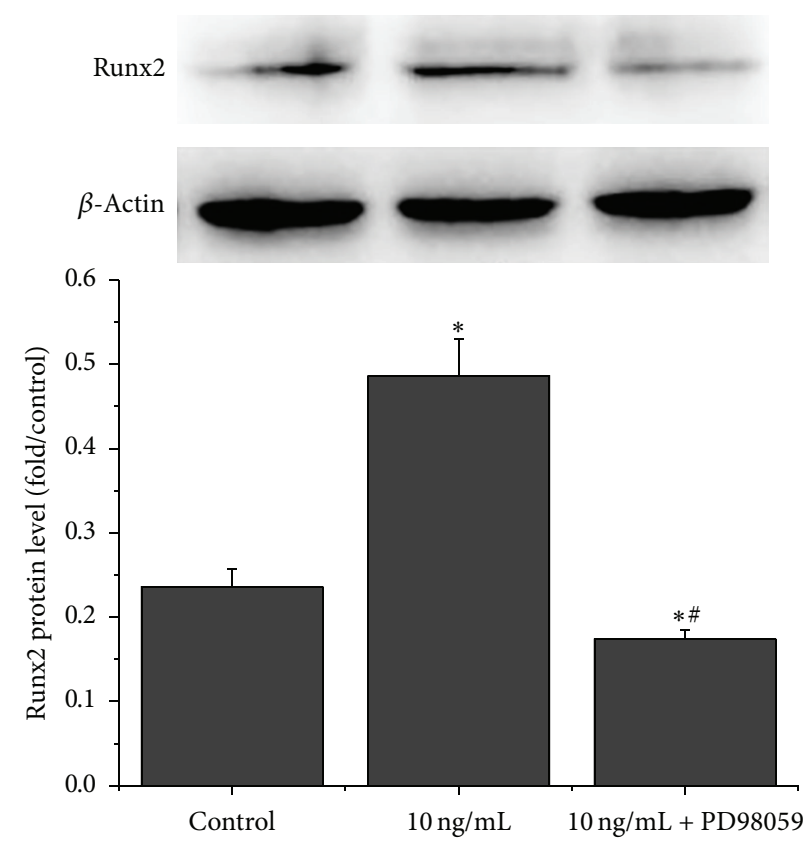

(b)

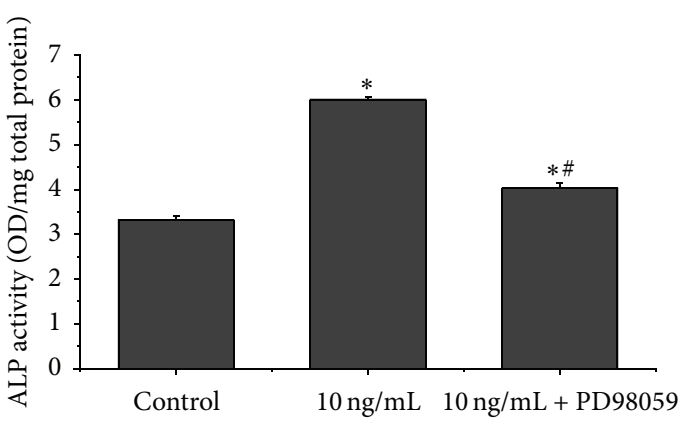

(d)

FIGURE 5: rhPDGF-BB-induced osteogenic differentiation in diabetic BMSCs was inhibited by suppression of ERK pathway. Cells were pretreated with or without $20 \mu \mathrm{M}$ PD98059 for $1 \mathrm{~h}$ and treated with $10 \mathrm{ng} / \mathrm{mL}$ rhPDGF-BB. The unmanipulated cells were used as controls. (a) Real-time PCR analysis of ALP, Runx2, and OCN mRNA expression on day 7. (b) Western blot analysis for the relative Runx2 protein levels on day 7. (c) ALP staining on day 14 (16x). (d) Quantitative results of ALP assay on day 14. (e) Alizarin red staining for mineralized nodules on day 21 (50x). ${ }^{*} P<0.05$, versus control, and ${ }^{\#} P<0.05$, versus $10 \mathrm{ng} / \mathrm{mL}$.

associated with diabetes such as poor bone defect healing and osseointegration.

We also performed studies to elucidate the mechanism of action of rhPDGF-BB on diabetic BMSCs. Our present findings showed that rhPDGF-BB directly enhanced the phosphorylation of ERK, consistent with observations in normal BMSCs [36]. It is well known that ERK pathway is crucial for regulation of cell proliferation, osteoblast differentiation, and skeletal development [37]. To demonstrate whether the induced effects of rhPDGF-BB on proliferation and osteoblast differentiation of diabetic BMSCs is dependent on ERK pathway, we used PD98059, a specific inhibitor of MEK. In this study, the results indicated that pretreatment with PD98059 blocked the rhPDGF-BB-induced ERK phosphorylation. Also, suspension of ERK by PD98059 inhibited rhPDGF-BB-stimulated enhanced proliferation in diabetic BMSCs. Moreover, PD98059 repressed rhPDGFBB-stimulated expressions of osteogenic genes (Runx2, ALP, 
and OCN) and Runx2 protein, as well as ALP activity and mineralization in diabetic BMSCs. These data suggested that rhPDGF-BB regulates proliferation and osteogenic differentiation of diabetic BMSCs partially through regulation of ERK phosphorylation. It has been well documented that ERK phosphorylation enhanced osteoblast differentiation, which has been attributed to the phosphorylation of Runx2 and regulating the transcriptional activity of Runx2 [38, 39]. Runx2 is a key and early transcription factor essential for osteoblast differentiation and bone formation, which could regulate ALP and OCN. As we observed that both the protein levels and the transcriptional activity of Runx2 were upregulated by rhPDGF-BB, we consider that rhPDGF-BB may stimulate osteogenic differentiation of diabetic BMSCs partially via inducing ERK-dependent Runx2 expression, which is needed to be further confirmed. In addition, as we previously found the inhibited ERK activation was correlated with the decreased osteogenic potential observed in the diabetic BMSCs compared with normal BMSCs [14]. It is plausible to propose that rhPDGF-BB could partially reverse the detrimental effect of diabetes on BMSCs and restore their partial functional impairment.

In summary, rhPDGF-BB could enhance cell proliferation and promote differentiation and mineralization in diabetic BMSCs. Furthermore, the enhanced osteogenic functions may be partially through ERK pathway. Although future studies are needed to further clarify whether rhPDGF$\mathrm{BB}$ induces the osteogenic ability of diabetic BMSCs in vivo, the present data indicate that rhPDGF-BB could be a potential therapeutic agent to partially restore proliferative and osteogenic differentiation impairments of diabetic BMSCs. And these results may suggest an effective strategy to employ the cell-based therapies in the treatment of challenging bone disorders in diabetes.

\section{Conflict of Interests}

The authors have no competing financial interests to declare.

\section{Acknowledgments}

The authors thank Xiuli Zhang, Wenjie Zhang, and Maolin Zhang for technical assistance. This work was jointly supported by National Basic Research Program of China (973 Program, no. 2012CB933604), National Science Fund for Distinguished Young Scholars of China (no. 81225006), and National Natural Science Foundation of China (no. 81170939 and no. 81201201).

\section{References}

[1] Y. Xu, L. Wang, J. He et al., "Prevalence and control of diabetes in Chinese adults," The Journal of the American Medical Association, vol. 310, no. 9, pp. 948-959, 2013.

[2] M. J. Silva, M. D. Brodt, M. A. Lynch et al., "Type 1 diabetes in young rats leads to progressive trabecular bone loss, cessation of cortical bone growth, and diminished whole bone strength and fatigue life," Journal of Bone and Mineral Research, vol. 24, no. 9, pp. 1618-1627, 2009.
[3] M. Janghorbani, R. M. van Dam, W. C. Willett, and F. B. Hu, "Systematic review of type 1 and type 2 diabetes mellitus and risk of fracture," The American Journal of Epidemiology, vol. 166, no. 5, pp. 495-505, 2007.

[4] S. Yaturu, "Diabetes and skeletal health," Journal of Diabetes, vol. 1, no. 4, pp. 246-254, 2009.

[5] L. Cozen, "Does diabetes delay fracture healing?" Clinical Orthopaedics and Related Research, vol. 82, pp. 134-140, 1972.

[6] A. M. Herskind, K. Christensen, K. Norgaard-Andersen, and J. F. Andersen, "Diabetes mellitus and healing of closed fractures," Diabete et Metabolisme, vol. 18, no. 1, pp. 63-64, 1992.

[7] P. Vestergaard, "Discrepancies in bone mineral density and fracture risk in patients with type 1 and type 2 diabetes-a metaanalysis," Osteoporosis International, vol. 18, no. 4, pp. 427-444, 2007.

[8] S. Harada and G. A. Rodan, "Control of osteoblast function and regulation of bone mass," Nature, vol. 423, no. 6937, pp. 349-355, 2003.

[9] L. C. Hofbauer, C. C. Brueck, S. K. Singh, and H. Dobnig, "Osteoporosis in patients with diabetes mellitus," Journal of Bone and Mineral Research, vol. 22, no. 9, pp. 1317-1328, 2007.

[10] L. R. McCabe, "Understanding the pathology and mechanisms of type I diabetic bone loss," Journal of Cellular Biochemistry, vol. 102, no. 6, pp. 1343-1357, 2007.

[11] A. Ogasawara, A. Nakajima, F. Nakajima, K. Goto, and M. Yamazaki, "Molecular basis for affected cartilage formation and bone union in fracture healing of the streptozotocin-induced diabetic rat," Bone, vol. 43, no. 5, pp. 832-839, 2008.

[12] S. Khosla, J. J. Westendorf, and U. I. Mödder, "Concise review: insights from normal bone remodeling and stem cell-based therapies for bone repair," Stem Cells, vol. 28, no. 12, pp. 21242128, 2010.

[13] J. R. Mauney, V. Volloch, and D. L. Kaplan, "Role of adult mesenchymal stem cells in bone tissue-engineering applications: current status and future prospects," Tissue Engineering, vol. 11, no. 5-6, pp. 787-802, 2005.

[14] Y. F. Zhao, D. L. Zeng, L. G. Xia et al., "Osteogenic potential of bone marrow stromal cells derived from streptozotocininduced diabetic rats," International Journal of Molecular Medicine, vol. 31, no. 3, pp. 614-620, 2013.

[15] A. Stolzing, D. Sellers, O. Llewelyn, and A. Scutt, "Diabetes induced changes in rat mesenchymal stem cells," Cells Tissues Organs, vol. 191, no. 6, pp. 453-465, 2010.

[16] J. O. Hollinger, C. E. Hart, S. N. Hirsch, S. Lynch, and G. E. Friedlaender, "Recombinant human platelet-derived growth factor: biology and clinical applications," Journal of Bone and Joint Surgery A, vol. 90, supplement 1, pp. 48-54, 2008.

[17] W. A. Tyndall, H. A. Beam, C. Zarro, J. P. O’Connor, and S. S. Lin, "Decreased platelet derived growth factor expression during fracture healing in diabetic animals," Clinical Orthopaedics and Related Research, no. 408, pp. 319-330, 2003.

[18] M. Nevins, W. V. Giannobile, M. K. McGuire et al., "Plateletderived growth factor stimulates bone fill and rate of attachment level gain: results of a large multicenter randomized controlled trial," Journal of Periodontology, vol. 76, no. 12, pp. 2205-2215, 2005.

[19] A. I. Caplan and D. Correa, "PDGF in bone formation and regeneration: new insights into a novel mechanism involving MSCs," Journal of Orthopaedic Research, vol. 29, no. 12, pp.17951803, 2011. 
[20] H. S. McCarthy, J. H. H. Williams, M. W. J. Davie, and M. J. Marshall, "Platelet-derived growth factor stimulates osteoprotegerin production in osteoblastic cells," Journal of Cellular Physiology, vol. 218, no. 2, pp. 350-354, 2009.

[21] J. O. Hollinger, A. O. Onikepe, J. MacKrell et al., "Accelerated fracture healing in the geriatric, osteoporotic rat with recombinant human platelet-derived growth factor-BB and an injectable $\beta$-tricalcium phosphate/collagen matrix," Journal of Orthopaedic Research, vol. 26, no. 1, pp. 83-90, 2008.

[22] L. Xu, K. Lv, W. Zhang, X. Zhang, X. Jiang, and F. Zhang, "The healing of critical-size calvarial bone defects in rat with rhPDGF-BB, BMSCs, and $\beta$-TCP scaffolds," Journal of Materials Science: Materials in Medicine, vol. 23, no. 4, pp. 1073-1084, 2012.

[23] L. Al-Zube, E. A. Breitbart, J. P. O'Connor et al., "Recombinant human platelet-derived growth factor BB (rhPDGF-BB) and $\beta$ tricalcium phosphate/collagen matrix enhance fracture healing in a diabetic rat model," Journal of Orthopaedic Research, vol. 27, no. 8, pp. 1074-1081, 2009.

[24] M. Retzepi, M. P. Lewis, and N. Donos, "Effect of diabetes and metabolic control on de novo bone formation following guided bone regeneration," Clinical Oral Implants Research, vol. 21, no. 1, pp. 71-79, 2010.

[25] K. Lü, D. Zeng, Y. Zhang et al., "BMP-2 gene modified canine bMSCs promote ectopic bone formation mediated by a nonviral PEI derivative," Annals of Biomedical Engineering, vol. 39, no. 6, pp. 1829-1839, 2011.

[26] X. Jiang, J. Zhao, S. Wang et al., "Mandibular repair in rats with premineralized silk scaffolds and BMP-2-modified bMSCs," Biomaterials, vol. 30, no. 27, pp. 4522-4532, 2009.

[27] M. Retzepi and N. Donos, "The effect of diabetes mellitus on osseous healing," Clinical Oral Implants Research, vol. 21, no. 7, pp. 673-681, 2010.

[28] E. A. Breitbart, S. Meade, V. Azad et al., "Mesenchymal stem cells accelerate bone allograft incorporation in the presence of diabetes mellitus," Journal of Orthopaedic Research, vol. 28, no. 7, pp. 942-949, 2010.

[29] M. Khan, S. Akhtar, S. Mohsin, S. N. Khan, and S. Riazuddin, "Growth factor preconditioning increases the function of diabetes-impaired mesenchymal stem cells," Stem Cells and Development, vol. 20, no. 1, pp. 67-75, 2011.

[30] C. Cramer, E. Freisinger, R. K. Jones et al., "Persistent high glucose concentrations alter the regenerative potential of mesenchymal stem cells," Stem Cells and Development, vol. 19, no. 12, pp. 1875-1884, 2010.

[31] M. Mezawa, S. Araki, H. Takai et al., "Regulation of human bone sialoprotein gene transcription by platelet-derived growth factor-BB," Gene, vol. 435, no. 1-2, pp. 80-87, 2009.

[32] T. Haque, H. Uludag, R. F. Zernicke, S. R. Winn, and W. Sebald, "Bone marrow cells from normal and ovariectomized rats respond differently to basic fibroblast growth factor and bone morphogenetic protein 2 treatment in vitro," Tissue Engineering, vol. 11, no. 3-4, pp. 634-644, 2005.

[33] M. Mehrotra, S. M. Krane, K. Walters, and C. Pilbeam, "Differential regulation of platelet-derived growth factor stimulated migration and proliferation in osteoblastic cells," Journal of Cellular Biochemistry, vol. 93, no. 4, pp. 741-752, 2004.

[34] R. T. Franceschi, C. Ge, G. Xiao, H. Roca, and D. Jiang, "Transcriptional regulation of osteoblasts," Annals of the New York Academy of Sciences, vol. 1116, pp. 196-207, 2007.

[35] T. Komori, "Regulation of skeletal development by the Runx family of transcription factors," Journal of Cellular Biochemistry, vol. 95 , no. 3, pp. 445-453, 2005.
[36] B. Gharibi, M. S. Ghuman, and F. J. Hughes, "Akt- and Erkmediated regulation of proliferation and differentiation during PDGFRbeta-induced MSC self-renewal," Journal of Cellular and Molecular Medicine, vol. 16, no. 11, pp. 2789-2801, 2012.

[37] C. Ge, G. Xiao, D. Jiang, and R. T. Franceschi, "Critical role of the extracellular signal-regulated kinase-MAPK pathway in osteoblast differentiation and skeletal development," Journal of Cell Biology, vol. 176, no. 5, pp. 709-718, 2007.

[38] G. Xiao, D. Jiang, P. Thomas et al., "MAPK pathways activate and phosphorylate the osteoblast-specific transcription factor, Cbfal," The Journal of Biological Chemistry, vol. 275, no. 6, pp. 4453-4459, 2000.

[39] C. Ge, Q. Yang, G. Zhao, H. Yu, K. L. Kirkwood, and R. T. Franceschi, "Interactions between extracellular signalregulated kinase 1/2 and P38 Map kinase pathways in the control of RUNX2 phosphorylation and transcriptional activity," Journal of Bone and Mineral Research, vol. 27, no. 3, pp. 538-551, 2012. 


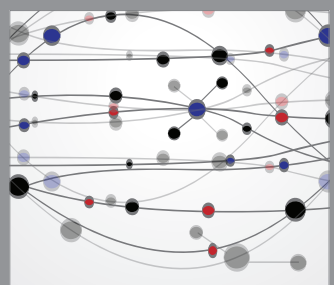

The Scientific World Journal
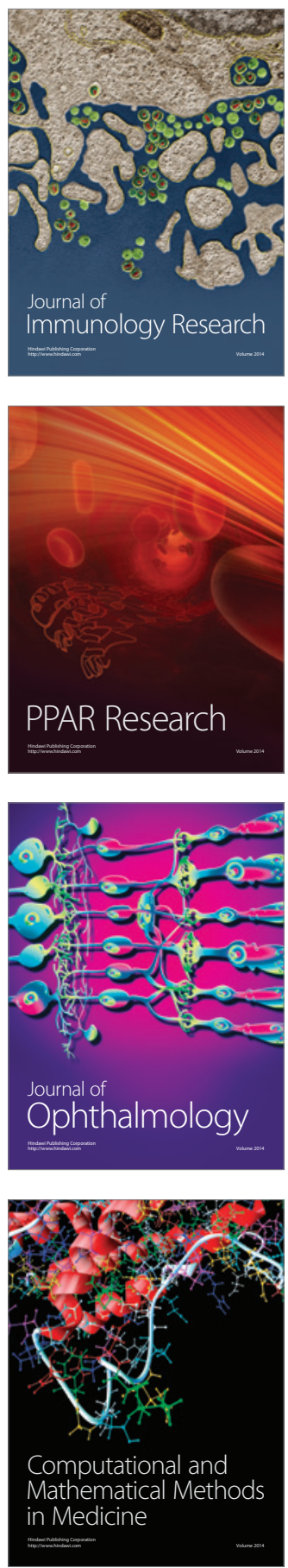

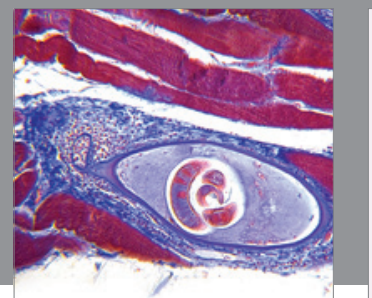

Gastroenterology

Research and Practice
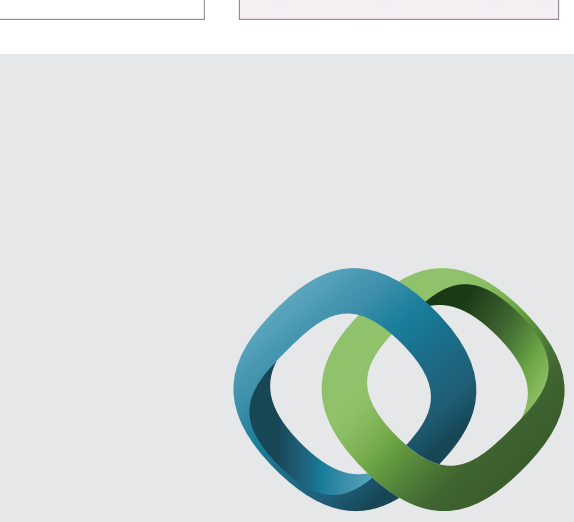

\section{Hindawi}

Submit your manuscripts at

http://www.hindawi.com
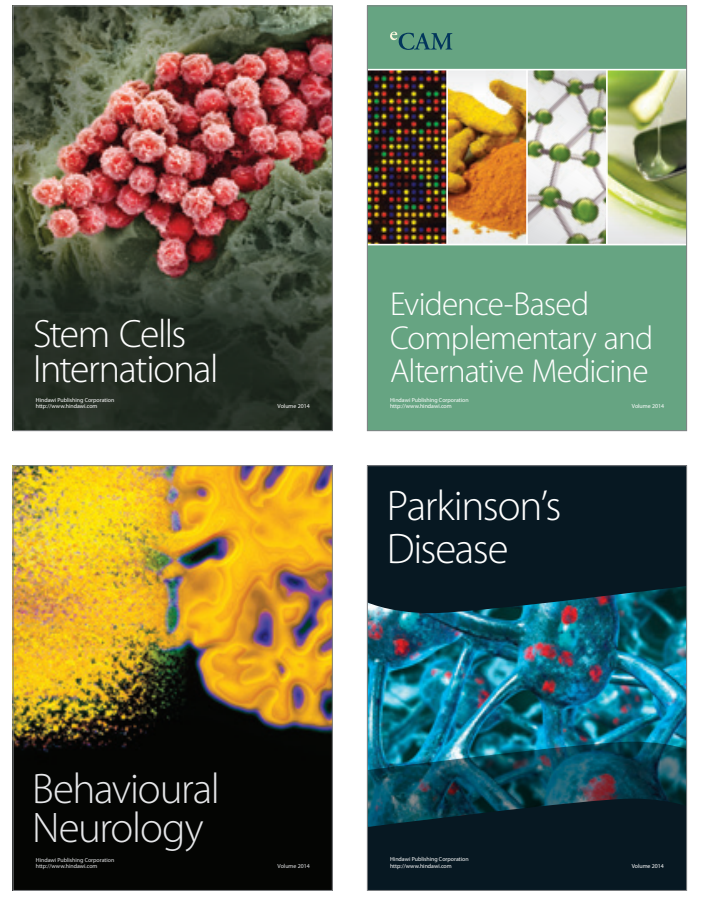
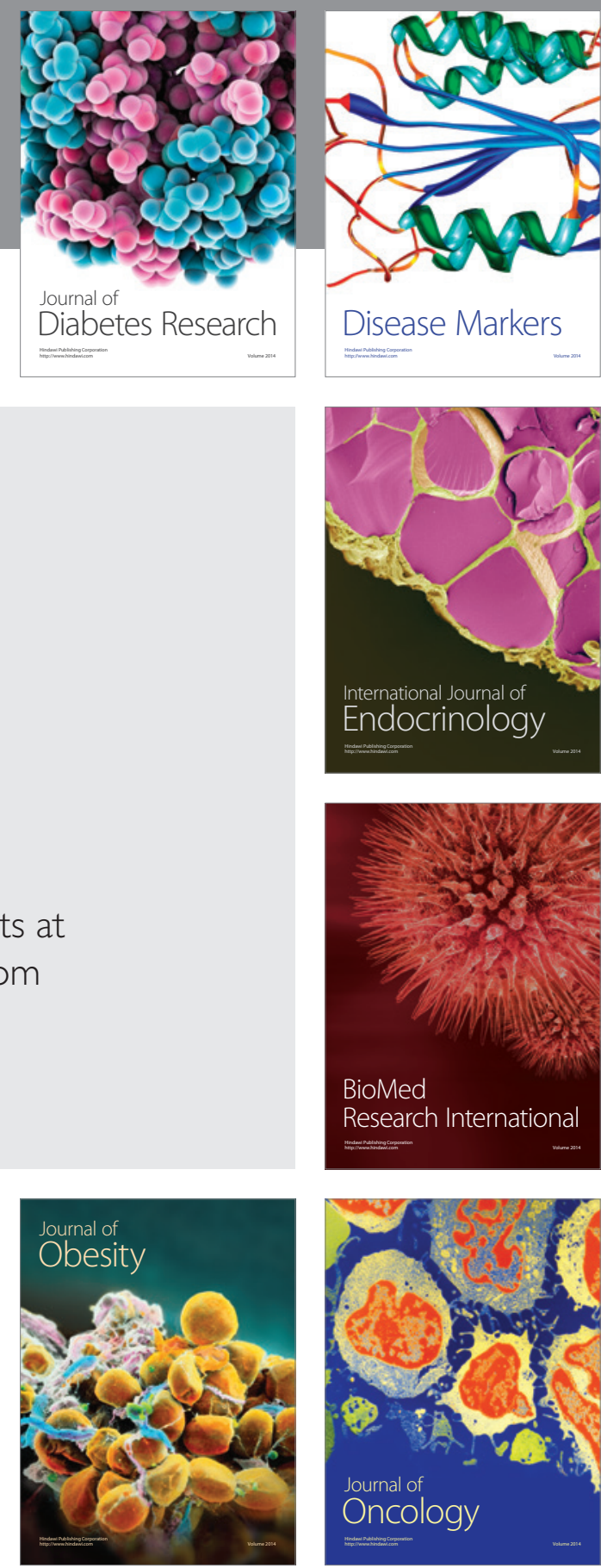

Disease Markers
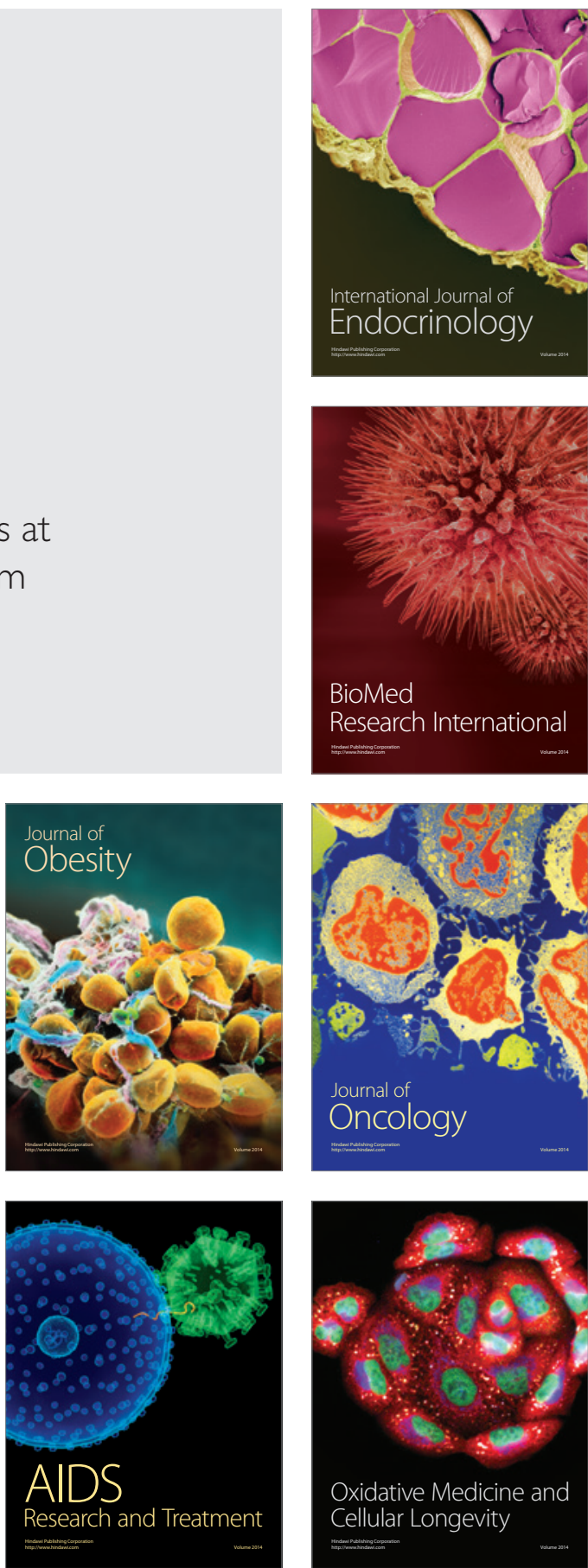